\title{
Evolution of multigap superconductivity in the atomically thin limit: Strain-enhanced three-gap superconductivity in monolayer $\mathrm{MgB}_{2}$
}

\author{
J. Bekaert, ${ }^{1, *}$ A. Aperis, ${ }^{2}$ B. Partoens, ${ }^{1}$ P. M. Oppeneer, ${ }^{2}$ and M. V. Miloševic ${ }^{1, \dagger}$ \\ ${ }^{1}$ Department of Physics, University of Antwerp, Groenenborgerlaan 171, B-2020 Antwerp, Belgium \\ ${ }^{2}$ Department of Physics and Astronomy, Uppsala University, Box 516, SE-751 20 Uppsala, Sweden \\ (Received 10 May 2017; revised manuscript received 18 July 2017; published 11 September 2017)
}

\begin{abstract}
Starting from first principles, we show the formation and evolution of superconducting gaps in $\mathrm{MgB}_{2}$ at its ultrathin limit. Atomically thin $\mathrm{MgB}_{2}$ is distinctly different from bulk $\mathrm{MgB}_{2}$ in that surface states become comparable in electronic density to the bulklike $\sigma$ and $\pi$ bands. Combining the ab initio electron-phonon coupling with the anisotropic Eliashberg equations, we show that monolayer $\mathrm{MgB}_{2}$ develops three distinct superconducting gaps, on completely separate parts of the Fermi surface due to the emergent surface contribution. These gaps hybridize nontrivially with every extra monolayer added to the film owing to the opening of additional coupling channels. Furthermore, we reveal that the three-gap superconductivity in monolayer $\mathrm{MgB}_{2}$ is robust over the entire temperature range that stretches up to a considerably high critical temperature of $20 \mathrm{~K}$. The latter can be boosted to $>50 \mathrm{~K}$ under biaxial tensile strain of $\sim 4 \%$, which is an enhancement that is stronger than in any other graphene-related superconductor known to date.
\end{abstract}

DOI: 10.1103/PhysRevB.96.094510

\section{INTRODUCTION}

A multigap superconductor is characterized by separate superconducting gaps opening on distinctly different parts of the Fermi surface [1]. The interest in this phenomenon and the emergent new physics was invigorated after the experimental discovery of two-gap superconductivity in bulk $\mathrm{MgB}_{2}$ in 2001 [2]. $\mathrm{MgB}_{2}$ consists of planes of boron in a honeycomb lattice alternated by planes of $\mathrm{Mg}$ atoms sitting above the centers of the honeycomb tiles. It is therefore akin to intercalated graphite [3], with $\mathrm{Mg}$ in the role of the dopant. In $\mathrm{MgB}_{2}$, in-plane $\sigma$ bonds coexist with out-of-plane $\pi$ bonds, and separately give rise to two superconducting gaps for bulk $\mathrm{MgB}_{2}$ : the stronger $\sigma$ gap, $\Delta_{\sigma}(0) \sim 7 \mathrm{meV}$, and the weaker $\pi$ gap, $\Delta_{\pi}(0) \sim 2-3 \mathrm{meV}$ [4-8].

Competition and coupling between the multiple condensates in a multigap superconductor can lead to rich new physics [9]. In that sense, one expects superconductors with three or more gaps to be far more exciting than the two-gap ones due to additional competing effects and possible quantum frustration between the condensates [10]. To date, the discovered effects specific to multigap superconductors include novel vortical and skyrmionic states $[11,12]$, giant-paramagnetic response [13], hidden criticality $[14,15]$, and time-reversal symmetry breaking $[10,16]$, to name a few. A major roadblock for the experimental confirmation of these predictions is the lack of distinctly multigap (beyond two-gap) superconductors. In recent years, two such materials were proposed theoretically by Gross and co-workers, using density functional theory for superconductors [17]. One is molecular hydrogen, which under very high pressure develops three superconducting gaps on different Fermi sheets [18]. However, due to anisotropy, two of the gaps strongly overlap. The other material is $\mathrm{CaBeSi}$, a $\mathrm{MgB}_{2}$-like compound in which splitting of the $\pi$ bands was

\footnotetext{
*jonas.bekaert@uantwerpen.be

†milorad.milosevic@uantwerpen.be
}

predicted to give rise to three-gap superconductivity [19], but with impractically low $T_{\mathrm{c}} \cong 0.4 \mathrm{~K}$.

Here, we follow a different route, namely, that of atomically thin, instead of bulk, superconductors. Recently, owing to immense experimental progress [20,21], superconductivity was realized down to monolayer thickness in several materialsranging from electron-phonon-based superconductors, such as $\mathrm{In}$ and $\mathrm{Pb}$ [22,23], $\mathrm{NbSe}_{2}$ [24-26], and doped graphene [27-32], to materials with nonconventional coupling mechanisms, such as $\mathrm{La}_{2-x} \mathrm{Sr}_{x} \mathrm{CuO}_{4}$ [33] and FeSe [34]. The promise for extremely low-power, ultralightweight, and ultrasensitive electronic devices warrants further progress in ultrathin superconductivity [35-37]. Quantum confinement in the vertical direction generally separates subbands in ultrathin films, inducing multiband and thereby potentially multigap superconductivity [38]. Here we note an additional, natural connection between two-dimensional and multigap superconductors, much less explored to date: surface states can equally host new superconducting gaps without any equivalent in the bulk material.

In this paper, we start from the known bulk two-gap superconductor $\mathrm{MgB}_{2}$ and show how the gap spectrum changes at the thinnest limit. It was predicted that, albeit not being the thermodynamic ground state, such structures are mechanically stable and could be grown owing to kinetic barriers [39], such that few-monolayer $\mathrm{MgB}_{2}$ has already been synthesized experimentally on a Mg substrate [40]. Using a combination of first-principles calculations and anisotropic Eliashberg theory, we reveal a major influence of an emerging surface state on superconductivity in these ultrathin films. This contribution hybridizes with those of the $\sigma$ and $\pi$ bands in a highly nontrivial manner, changing the multigap physics with every additional monolayer. This finally leads to pure three-gap superconductivity in one-monolayer $\mathrm{MgB}_{2}$, retained up to a high critical temperature of $20 \mathrm{~K}$ (highest among monolayer superconductors without coupling to a substrate). This superconductivity originating from the surface state could not be detected by a previous study of few-monolayer 
$\mathrm{MgB}_{2}$ based on the tight-binding formalism, in which surface states (electronic as well as vibrational) were completely omitted [41]. We further demonstrate that this three-gap superconductivity remains robust even under strain, where tensile strain of just $\sim 4 \%$ boosts $T_{\mathrm{c}}$ to above $50 \mathrm{~K}$. Such small strain was previously found to increase $T_{\mathrm{c}}$ in bulk $\mathrm{MgB}_{2}$ by, at most, $10 \%[42,43]$ or nearly not at all in both electron-doped [44] and hole-doped [45,46] graphene (only strain beyond 5\% is predicted to have significant influence there). Considering that such straining can be conveniently realized by growing the monolayer $\mathrm{MgB}_{2}$ on substrates with a somewhat larger lattice constant (e.g., $\mathrm{Si}_{1+x} \mathrm{C}_{1-x}$ or $\mathrm{Al}_{x} \mathrm{Ga}_{1-x} \mathrm{~N}$ alloys, with a lattice constant tunable by $x$ ) [43], we expect our results to be of immediate experimental relevance.

\section{MONOLAYER MgB}

Our investigation starts from first-principles calculations (using ABINIT [47]; see also the Appendices) of one monolayer (ML) of $\mathrm{MgB}_{2}$. It consists of one $\mathrm{Mg}$ and one $\mathrm{B}$ layer, with the latter in a honeycomb lattice and thus structurally similar to doped graphene. The resulting Fermi surface is shown in Fig. 1(a). It consists of two $\sigma$ bands (around $\Gamma$ ), a $\pi$ band (around $\mathrm{K}$ ), and a surface band $S$. While, as we mentioned above, the former two are also present in bulk $\mathrm{MgB}_{2}$, the surface band originates from the $\mathrm{Mg}$ plane facing vacuum. It is thus characteristic of two-dimensional forms of $\mathrm{MgB}_{2}$ and has predominant $\mathrm{Mg}$ - $p$ character, as opposed to the $\mathrm{B}-p$ character of the other bands. Next, we calculated the electron-phonon (eph) coupling in one-ML $\mathrm{MgB}_{2}$ from first principles, employing density functional perturbation theory (DFPT) [47,48]. With this input, the anisotropic Eliashberg equations (i.e., taking into account the full spatial dependence) were solved selfconsistently $[8,49]$ (see Appendices).

We describe the Coulomb repulsion with $\mu^{*}=0.13$, yielding correct $T_{\mathrm{c}}$ for bulk $\mathrm{MgB}_{2}$. This value is also in line with previously established values $[5,50]$. The Coulomb pseudopotential is not expected to change drastically in the two-dimensional limit, owing to the layered structure of $\mathrm{MgB}_{2}$. Namely, superconductivity of the dominant $\sigma$ bands is quasi-two-dimensional even in bulk $\mathrm{MgB}_{2}$, so the same is expected for the screening.

In Fig. 1(a), we show the resulting superconducting gap spectrum on the Fermi surface, $\Delta\left(\mathbf{k}_{\mathrm{F}}, T\right)$, at $T=1 \mathrm{~K}$, as well as the distribution of the gap, $\rho(\Delta)$. This result shows that one-ML $\mathrm{MgB}_{2}$ is a distinctly three-gap superconductor, with separate gaps opening on the $\sigma, \pi$, and $S$ bands. The gap amplitudes are about half of those of bulk $\mathrm{MgB}_{2}$, with Fermi surface averages at zero temperature of $\left\langle\Delta_{\sigma}(0)\right\rangle=3.3 \mathrm{meV}$, $\left\langle\Delta_{S}(0)\right\rangle=2.7 \mathrm{meV}$, and $\left\langle\Delta_{\pi}(0)\right\rangle=1.4 \mathrm{meV}$. The critical temperature of $T_{\mathrm{c}}=20 \mathrm{~K}$, compared to the bulk $T_{\mathrm{c}} \cong 39 \mathrm{~K}$ [4-8], follows the same trend.

To further corroborate the predicted three-gap superconductivity in one-ML $\mathrm{MgB}_{2}$, we calculated the density of states (DOS) in the superconducting state $N_{\mathrm{S}}$, using Eliashberg relations [8] (see Appendices). The result displayed in Fig. 1(b) shows that $N_{\mathrm{S}}$ for one-ML $\mathrm{MgB}_{2}$ consists of three distinct and narrow peaks, corresponding to the three superconducting gaps. As $N_{\mathrm{S}}$ determines the superconducting tunneling properties, the predicted three-gap superconductivity can be verified with low-temperature scanning tunneling spectroscopy [4].

Last but not least, we show that three-gap superconductivity in one-ML $\mathrm{MgB}_{2}$ is very robust with temperature. Figure 1(c) displays the calculated temperature evolution of the superconducting gap spectrum, proving that the three superconducting gaps are well separated up to $18 \mathrm{~K}$, very close to $T_{\mathrm{c}}=20 \mathrm{~K}$.

\section{EVOLUTION WITH ADDED MONOLAYERS}

To provide a deeper understanding of the origin of three-gap superconductivity in one-ML $\mathrm{MgB}_{2}$, we studied what changes when adding monolayers to the system, considering in particular two- and four-ML-thick $\mathrm{MgB}_{2}$. The superconducting gap spectra, obtained using anisotropic Eliashberg theory, are displayed in Figs. 2(a) and 2(b). One observes in Fig. 2(a) that a hexagonal band lying between the $S$ band and the $\sigma$ bands develops an additional gap in two-ML $\mathrm{MgB}_{2}$. This band is a split-off band of the $\sigma$ bands (with B- $p$ character), indicated with $S^{\prime}$ as it originates from a surface state of the free B surface. The superconducting gap opening on band $S$, is weakly linked to the gaps opening on the $\pi$ and $S$ bands,
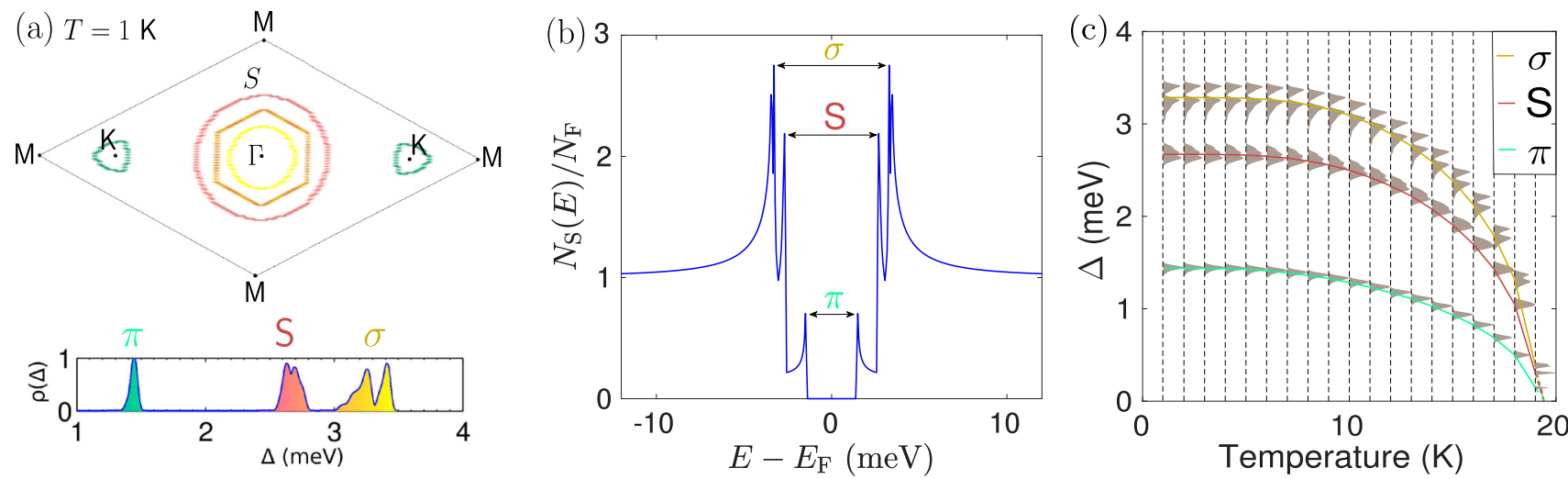

FIG. 1. The superconducting spectrum of one-ML $\mathrm{MgB}_{2}$, calculated by anisotropic Eliashberg theory with $a b$ initio input. (a) The distribution of the three superconducting gaps $\Delta\left(\mathbf{k}_{\mathrm{F}}, T\right)$ on the Fermi surface: $\pi, S$ (for surface), and $\sigma$, at $T=1 \mathrm{~K}$. (b) The density of states in the superconducting state at $T=1 \mathrm{~K}$, showing three distinct peaks corresponding to the three gaps. (c) The evolution of the gap spectrum with temperature, including the gap averages. The calculation shows that one-ML $\mathrm{MgB}_{2}$ has $T_{\mathrm{c}} \cong 20 \mathrm{~K}$. 

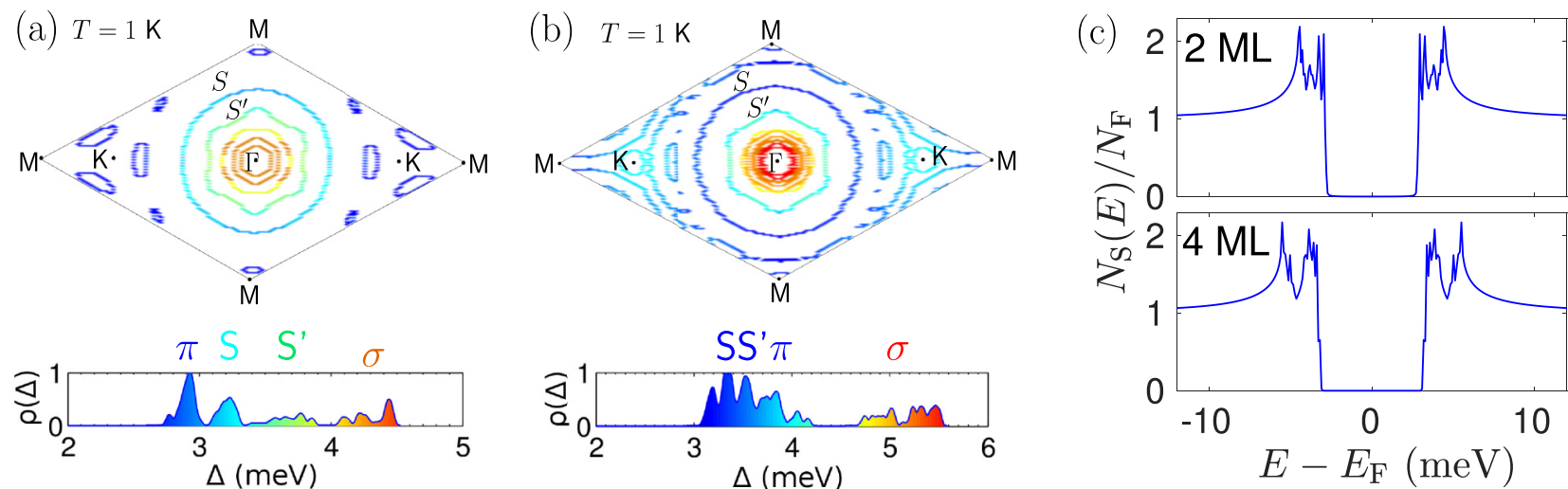

FIG. 2. (a),(b) The distribution of the superconducting gap spectrum of two-ML and four-ML $\mathrm{MgB}_{2}$, respectively, on the Fermi surface, calculated from anisotropic Eliashberg theory with $a b$ initio input. Both are anisotropic two-gap superconductors, with surface condensates $S$ and $S$ ' hybridized with the $\pi$ condensate. (c) The density of states in the superconducting state for two and four MLs, calculated at $T=1 \mathrm{~K}$, showing the overall two-gap nature as well as the anisotropy of the gap spectrum. The critical temperatures found for two- and four-ML $\mathrm{MgB}_{2}$ are $23 \mathrm{~K}$ and $27 \mathrm{~K}$, respectively.

but (barely) separate from the gap on the $\sigma$ bands, making two-ML $\mathrm{MgB}_{2}$ an anisotropic two-gap (nearly single-gap) superconductor. In four-ML $\mathrm{MgB}_{2}$, we find a higher degree of hybridization between the $\pi, S$, and $S^{\prime}$ condensates, forming an anisotropic gap clearly separated from the $\sigma$ gap. In Fig. 2(c), we show the corresponding DOS in the superconducting state. For two-ML $\mathrm{MgB}_{2}, N_{\mathrm{S}}$ clearly reflects the anisotropy of the gap spectrum, while for four-ML $\mathrm{MgB}_{2}, N_{\mathrm{S}}$ consists of two broader peaks, resulting from the strong hybridization between the condensates. The critical temperatures we obtained from the solution of the anisotropic Eliashberg equations are larger than that of one-ML $\mathrm{MgB}_{2}$, namely $23 \mathrm{~K}$ and $27 \mathrm{~K}$ for two-ML and four-ML $\mathrm{MgB}_{2}$, respectively (still well below the bulk value of $39 \mathrm{~K}$ [51]).

The transition from three-gap superconductivity in ML $\mathrm{MgB}_{2}$ to anisotropic two-gap superconductivity and two-ML and four-ML $\mathrm{MgB}_{2}$ can be explained by means of the e-ph coupling field shown in Fig. 3. In all cases, the e-ph coupling peaks for phonon wave vectors $\mathbf{q} \simeq 0$ (i.e., $\Gamma$ ), which promotes intraband coupling, giving rise to separate condensates on different sheets. However, in Fig. 3, one also observes a clear evolution towards stronger coupling at nonzero wave vectors going from a ML to thicker structures. These emerging coupling channels enable scattering between different sheets, notably between the close-lying $S, S^{\prime}$, and $\pi$ bands. This leads to the hybridization between the corresponding condensates shown in Fig. 2.

Our results show thus a drastic change from the distinctly three-gap superconductivity in single-ML $\mathrm{MgB}_{2}$ to very anisotropic two-gap superconductivity by the addition of even a single monolayer. Bearing in mind that the superconducting gap opening on the surface band in very thick $\mathrm{MgB}_{2}$ films was found experimentally to be nearly degenerate with the gap on the $\sigma$ band [52], we expect further rich behavior of the gap spectrum as the $\mathrm{MgB}_{2}$ film is made progressively thicker beyond four MLs. Besides the accompanying fundamental physics, this strong variation of the gap structure with the number of MLs opens perspectives for nanoengineered superconducting junctions using one single material with spatially varied thickness on the atomic scale. Such local control of thickness is readily available for, e.g., $\mathrm{Pb}$ films [20,21].

\section{STRAINED MONOLAYER MgB}

In experiments, the preferred growth method of atomically thin $\mathrm{MgB}_{2}$ is epitaxial growth on a substrate [40]. Due to the ever-present lattice mismatch in that case, we consider the effect of strain on the three-gap superconductivity predicted here. We concentrate on biaxial strain applied with respect to

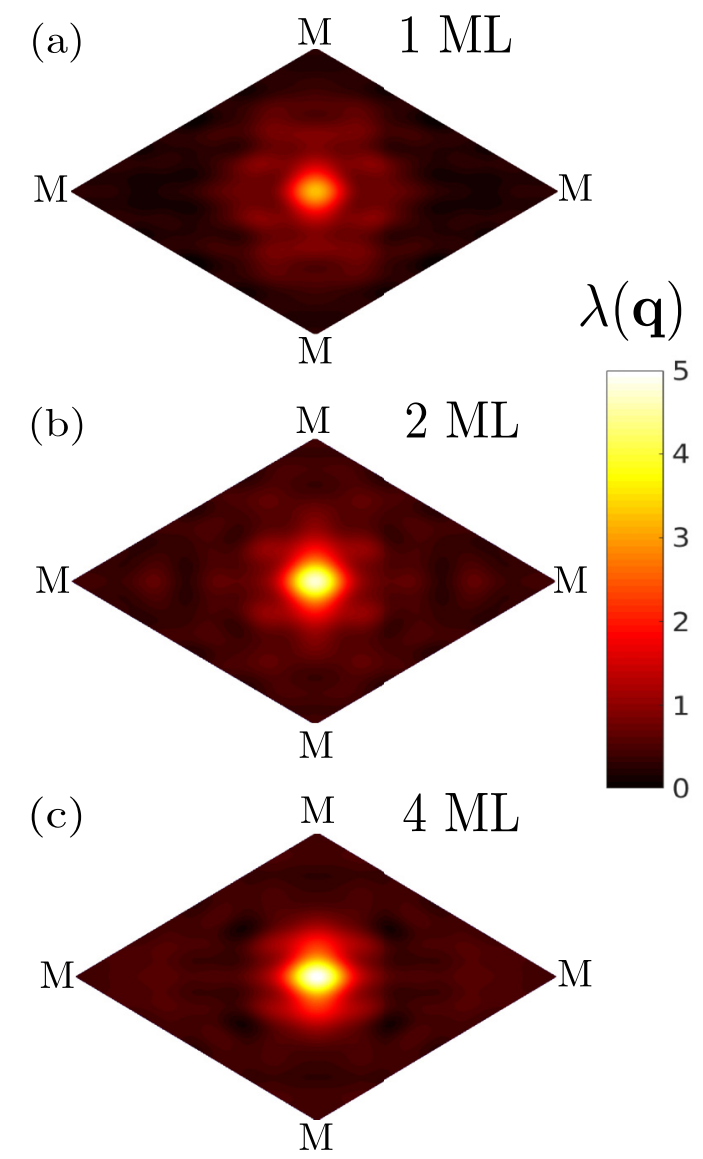

FIG. 3. The overall e-ph coupling $\lambda(\mathbf{q})=\sum_{v} \lambda_{v}(\mathbf{q})$ (i.e., summed over all phonon modes) as a function of phonon wave vectors $\mathbf{q}$ for (a) one-ML, (b) two-ML, and (c) four-ML $\mathrm{MgB}_{2}$. 

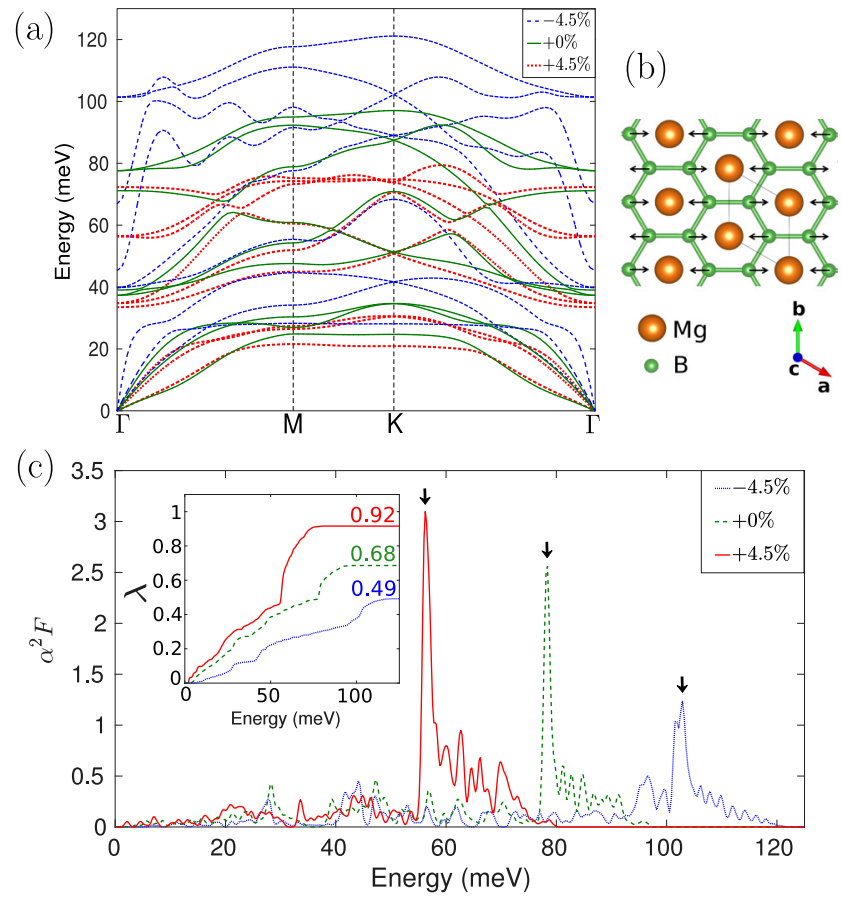

FIG. 4. Phonons and electron-phonon coupling of biaxially strained one-ML $\mathrm{MgB}_{2}$ calculated using DFPT. (a) The phonon dispersion for strains of $-4.5 \%,+0 \%$, and $+4.5 \%$. Increasing strain leads to lower phonon frequencies. (b) The $E_{2 g}$ phonon mode of the $\mathrm{B}$ atoms that gives the strongest contribution to the electron-phonon coupling. (c) The isotropic Eliashberg function under different strains, $\alpha^{2} F(\omega)=\left\langle\left\langle\alpha^{2} F\left(\mathbf{k} \mathbf{k}^{\prime}, \omega\right)\right\rangle_{\mathbf{k}_{\mathrm{F}}^{\prime}}\right\rangle_{\mathbf{k}_{\mathrm{F}}}$ (i.e., the double Fermi surface average). The peaks originating from the $E_{2 g}$ mode are indicated by arrows. The resulting electron-phonon coupling $\lambda$ is shown as the inset.

the in-plane cell parameter, namely, the $\mathrm{Mg}-\mathrm{Mg}$ distance with equilibrium value $a=3.04 \AA$. In Fig. 4(a), we compare the equilibrium phonon band structure of one-ML $\mathrm{MgB}_{2}$ with the cases of $-4.5 \%$ compressive strain and $+4.5 \%$ tensile strain. In the tensile case, interatomic charge densities get depleted as the distances between atoms increase. Consequently, the interatomic bonds become less stiff, resulting in a decrease of phonon frequencies. In the compressive case, the exact opposite occurs. In Fig. 4(b), we show the $E_{2 g}$ phonon mode of the $\mathrm{B}$ atoms, which is the mode harboring the strongest e-ph coupling in one-ML $\mathrm{MgB}_{2}$. As such, this mode dominates the Eliashberg function $\alpha^{2} F$, shown in Fig. 4(c). The peaks in $\alpha^{2} F$ due to the $E_{2 g}$ mode (indicated by arrows) are stronger and more pronounced in one-ML $\mathrm{MgB}_{2}$ compared with bulk $\mathrm{MgB}_{2}$ [8], in particular in equilibrium and under tensile strain. The shift to lower energy (following the general trend for the phonons) and amplification of this peak due to tensile strain lead to a significant enhancement of the e-ph coupling, as shown in the inset of Fig. 4(c). As follows from the above discussion, it is a general principle that tensile strain lowers the energy of the phonon modes, resulting in enhanced e-ph coupling, since $\lambda=2 \int_{0}^{\infty} d \omega \omega^{-1} \alpha^{2} F(\omega)$ is weighted by $\omega^{-1}$ [53]. However, the effect is particularly strong in one-ML $\mathrm{MgB}_{2}$ due to the occurrence of the $E_{2 g}$ phonon mode, which not only goes down in energy but also develops stronger
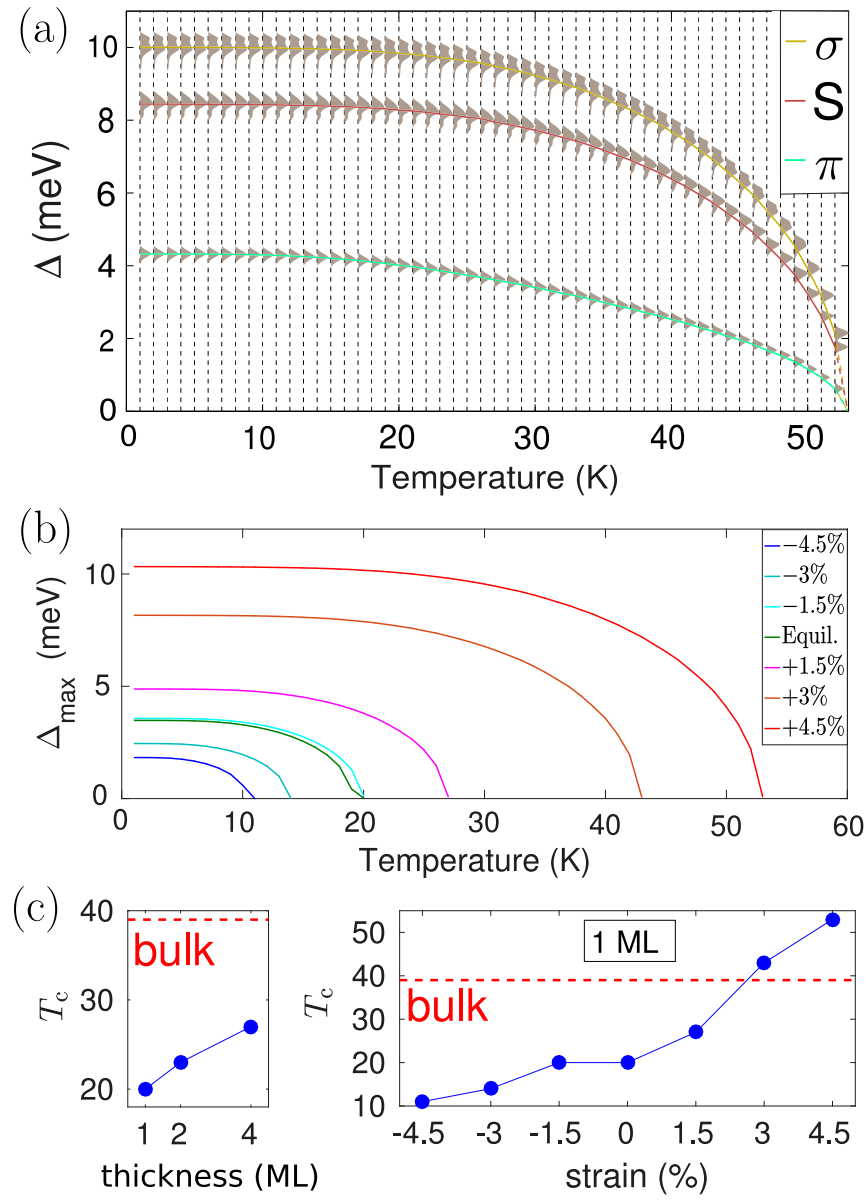

FIG. 5. The superconducting spectrum of a biaxially strained one-ML $\mathrm{MgB}_{2}$. (a) The distribution of the superconducting gap for $+4.5 \%$ tensile strain as a function of temperature, displaying the same three gaps ( $\pi, S$, and $\sigma$ ) as in the unstrained case (Fig. 1). The calculation shows an enhancement of the critical temperature to $T_{\mathrm{c}}=53 \mathrm{~K}$. (b) The maximum value of the superconducting gap, $\Delta_{\max }$, as a function of temperature and strain. Superconductivity depletes upon compression and is strongly boosted with tensile strain. (c) $T_{\mathrm{c}}$ as a function of the film thickness and as a function of strain for a one-ML $\mathrm{MgB}_{2}$. The bulk value, $T_{\mathrm{c}}=39 \mathrm{~K}$, is shown for comparison.

intrinsic coupling to electrons, as follows from the evolution of the Eliashberg function shown in Fig. 4(c). A similar trend in the e-ph coupling under the influence of strain has been found in both electron- and hole-doped graphene [44-46], although much less pronounced.

With this first-principles input for strained one-ML $\mathrm{MgB}_{2}$, we solved again the anisotropic Eliashberg equations. We found that the Fermi surface is almost unaltered with respect to that shown in Fig. 1(a), in the studied range of straining of $-4.5 \%$ to $+4.5 \%$. This, in combination with the robust coupling to the $E_{2 g}$ mode, leads to three-gap superconductivity in $\mathrm{ML} \mathrm{MgB}_{2}$ being conserved under all strains considered here [54]. In Fig. 5(a), we show the temperature evolution of the gap spectrum of one-ML $\mathrm{MgB}_{2}$ subject to tensile strain of $+4.5 \%$, proving the robustness of the three-gap superconductivity even under a considerable amount of strain. Owing to the enhanced e-ph coupling [cf. Fig. 4(c)], the superconducting gaps are much larger than in the equilibrium 
case. For $+4.5 \%$ strain, the average gaps amount to $\left\langle\Delta_{\sigma}(0)\right\rangle=$ $10.0 \mathrm{meV},\left\langle\Delta_{S}(0)\right\rangle=8.4 \mathrm{meV}$, and $\left\langle\Delta_{\pi}(0)\right\rangle=4.3 \mathrm{meV}$, with a corresponding critical temperature as high as $T_{\mathrm{c}}=53 \mathrm{~K}$. In Fig. 5(b), we show the temperature evolution of the maximum $(\sigma)$ gap value, comparatively for different strains. It reveals that upon compression, superconductivity is greatly suppressed ( $T_{\mathrm{c}}$ drops to $11 \mathrm{~K}$ for $-4.5 \%$ strain), while it is strongly boosted when the ML is subject to tensile strain. The changes are particularly drastic for such limited amounts of strain, in comparison to, e.g., superconducting doped graphene [44-46]. In Fig. 4(c), we show the evolution of $T_{\mathrm{c}}$ with the number of monolayers and with strain. It is apparent that the effect of strain on superconductivity is stronger, with a ML strained at $+3 \%$ already surpassing bulk $\mathrm{MgB}_{2}$ as to its $T_{\mathrm{c}}$. A major difference between both manipulations we considered is that strain preserves the three-gap superconductivity of monolayer $\mathrm{MgB}_{2}$, while increasing thickness strongly changes the gap spectrum with every added monolayer, as shown in Fig. 2.

\section{CONCLUSION}

In summary, we presented the formation and evolution of three-gap superconductivity in few-monolayer $\mathrm{MgB}_{2}$, by solving the anisotropic Eliashberg equations with full $a b$ initio input. We showed that the electronic surface band, originating from the free $\mathrm{Mg}$ surface, plays a major role in ultrathin $\mathrm{MgB}_{2}$ and hosts a third superconducting gap that coexists with the bulklike $\pi$ and $\sigma$ gaps. These gaps are distinctly separate in one-monolayer $\mathrm{MgB}_{2}$, where the resulting three pronounced peaks in the superconducting tunneling spectrum provide a clear signature for experimental validation of our prediction. The shown three-gap superconductivity is moreover very robust with temperature, persisting even close to the critical temperature of $20 \mathrm{~K}$. With only $\sim 4 \%$ tensile strain, the electron-phonon coupling is greatly enhanced and superconductivity is boosted to temperatures beyond $50 \mathrm{~K}$. As more monolayers are added to the film, different condensates hybridize, changing the multigap spectrum drastically with every added monolayer. Our investigation therefore establishes atomically thin $\mathrm{MgB}_{2}$ as a unique system to explore tunability of high- $T_{\mathrm{c}}$, multigap superconductivity, and its possible applications in ultrathin cryogenic electronics engineered by strain and atomically controlled thickness.

\section{ACKNOWLEDGMENTS}

This work was supported by TOPBOF-UAntwerp, Research Foundation-Flanders (FWO), the Swedish Research Council (VR), and the Röntgen-Ångström Cluster. The first-principles calculations have been carried out on the HPC infrastructure of the University of Antwerp (CalcUA), a division of the Flemish Supercomputer Centre (VSC), supported financially by the Hercules Foundation and the Flemish Government (EWI Department). Eliashberg theory calculations were supported through the Swedish National Infrastructure for Computing (SNIC).

\section{APPENDIX A: DENSITY FUNCTIONAL (PERTURBATION) THEORY CALCULATIONS}

Our density functional theory (DFT) calculations make use of the Perdew-Burke-Ernzerhof (PBE) functional implemented within a plane-wave basis in the ABINIT code [47]. Electron-ion interactions are treated using norm-conserving Vanderbilt pseudopotentials [55], taking into account $\mathrm{Mg}$ $2 s^{2} 2 p^{6} 3 s^{2}$ and B-2 $s^{2} 2 p^{1}$ as valence electrons. An energy cutoff of $60 \mathrm{Ha}$ for the plane-wave basis was used to achieve convergence of the total energy below $1 \mathrm{meV}$ per atom. In order to simulate the atomically thin films, we used unit cells that include $25 \AA$ of vacuum. A dense $22 \times 22 \times 1 \Gamma$ centered Monkhorst-Pack k-point grid is used for an accurate description of the Fermi surfaces. The lattice parameters were obtained using a conjugate-gradient algorithm so that forces on each atom were minimized below $1 \mathrm{meV} / \AA$. Strain was implemented by changing the in-plane lattice parameter with respect to the equilibrium value thus obtained.

To calculate phonon dispersions and electron-phonon coupling, density functional perturbation theory (DFPT) calculations were carried out, also within the framework of ABINIT. The total number of perturbations due to atomic displacements to be treated (in other words, the number of phonon branches) amounts to $3 N_{\text {atoms }}$, ranging from 9 for one ML to 36 for four MLs. Thus, the phonon spectrum and electron-phonon coupling coefficients, matrix elements of the perturbative part of the Hamiltonian [48], are obtained. We carried out the DFPT calculations on a $22 \times 22 \times 1$ electronic k-point grid and a $11 \times 11 \times 1 \mathbf{q}$-point grid (a subgrid of the $\mathbf{k}$-point grid) as phonon wave vectors.

\section{APPENDIX B: FULLY ANISOTROPIC ELIASHBERG THEORY CALCULATIONS}

In order to describe superconductivity of $\mathrm{MgB}_{2}$ on an $a b$ initio level, we self-consistently solve the coupled anisotropic Eliashberg equations [5,8],

$$
\begin{aligned}
Z_{\mathbf{k}, n}= & 1+\frac{\pi T}{\omega_{n}} \sum_{\mathbf{k}^{\prime}, n^{\prime}} \frac{\delta\left(\xi_{\mathbf{k}^{\prime}}\right)}{N_{F}} \lambda\left(\mathbf{k k}^{\prime}, n n^{\prime}\right) \\
& \times \frac{\omega_{n^{\prime}}}{\sqrt{\omega_{n^{\prime}}^{2}+\Delta_{\mathbf{k}^{\prime}, n}^{2}}}, \\
\Delta_{\mathbf{k}, n} Z_{\mathbf{k}, n}= & \pi T \sum_{\mathbf{k}^{\prime}, n^{\prime}} \frac{\delta\left(\xi_{\mathbf{k}^{\prime}}\right)}{N_{F}}\left[\lambda\left(\mathbf{k} \mathbf{k}^{\prime}, n n^{\prime}\right)-\mu^{*}\left(\omega_{c}\right)\right] \\
& \times \frac{\Delta_{\mathbf{k}^{\prime}, n^{\prime}}}{\sqrt{\omega_{n^{\prime}}^{2}+\Delta_{\mathbf{k}, n}^{2}}},
\end{aligned}
$$

using the ab initio calculated electron band structure contained in $\xi_{\mathbf{k}}$ and phonon and electron-phonon coupling contained in $\lambda\left(\mathbf{k} \mathbf{k}^{\prime}, n n^{\prime}\right)$. In the above, $T$ is temperature, $\omega_{n}=\pi T(2 n+1)$ are fermion Matsubara frequencies, $Z_{\mathbf{k}, n}$ is the mass renormalization function, $\Delta_{\mathbf{k}, n}$ describes anisotropic even-frequency spin-singlet superconductivity, $N_{\mathrm{F}}$ is the electronic density of states at the Fermi level, and $\mu^{*}\left(\omega_{c}\right)$ is the Anderson-Morel Coulomb pseudopotential which comes with a cutoff $\omega_{c}$. The 
momentum-dependent electron-phonon coupling is

$$
\lambda\left(\mathbf{k}-\mathbf{k}^{\prime}, n-n^{\prime}\right)=\int_{0}^{\infty} d \omega \alpha^{2} F\left(\mathbf{k} \mathbf{k}^{\prime}, \omega\right) \frac{2 \omega}{\left(\omega_{n}-\omega_{n^{\prime}}\right)^{2}+\omega^{2}},
$$

with the momentum-dependent Eliashberg function

$$
\alpha^{2} F\left(\mathbf{k} \mathbf{k}^{\prime}, \omega\right)=N_{F} \sum_{\nu}\left|g_{\mathbf{q}}^{v}\right|^{2} \delta\left(\omega-\omega_{\mathbf{q} v}\right)
$$

where $\mathbf{q}=\mathbf{k}-\mathbf{k}^{\prime}$ and where $g_{\mathbf{q}}^{\nu}$ and $\omega_{\mathbf{q} v}$ are the phonon branch-resolved electron-phonon scattering matrix elements and phonon frequencies, respectively. From the above, one can obtain the isotropic Eliashberg function as

$$
\alpha^{2} F(\omega)=\left\langle\left\langle\alpha^{2} F\left(\mathbf{k} \mathbf{k}^{\prime}, \omega\right)\right\rangle_{\mathbf{k}_{\mathrm{F}}^{\prime}}\right\rangle_{\mathbf{k}_{\mathrm{F}}},
$$

where $\langle\cdot\rangle_{\mathbf{k}_{\mathrm{F}}}=\frac{1}{N_{\mathrm{F}}} \sum_{\mathbf{k}} \delta\left(\xi_{\mathbf{k}}\right)(\ldots)$ is the Fermi surface average.

The quasiparticle density of states that is proportional to single-particle tunneling measurements is given by

$$
N_{\mathrm{S}}(\Omega) \propto \sum_{\mathbf{k}} A(\mathbf{k}, \Omega) \approx N_{\mathrm{F}}\left\langle\int_{-\infty}^{\infty} d \xi A_{\mathbf{k}}(\xi, \Omega)\right\rangle_{\mathbf{k}_{\mathrm{F}}},
$$

with the spectral function

$$
A(\mathbf{k}, \Omega)=-\frac{1}{\pi} \operatorname{Im}\left[\hat{G}_{R}(\mathbf{k}, \Omega)\right]_{11},
$$

where $\left[\hat{G}_{R}(\mathbf{k}, \Omega)\right]_{11}$ is the (11) element of the retarded matrix Green's function, obtained after analytic continuation of the full matrix Green's function,

$$
\hat{G}_{\mathbf{k}, n}=\left[i \omega_{n} Z_{\mathbf{k}, n} \hat{\rho}_{0}-\xi_{\mathbf{k}} \hat{\rho}_{3}-\Delta_{\mathbf{k}, n} \hat{\rho}_{1}\right]^{-1}
$$

The coupled equations (B1),(B2), supplemented by the electron and phonon band structure and the electron-phonon coupling, calculated by first principles, were solved selfconsistently in Matsubara space and the converged solutions were then analytically continued to real frequencies. In order to ensure a good accuracy, we imposed a strict convergence criterion of $\frac{x_{n}-x_{n-1}}{x_{n}}<10^{-6}$ and allowed up to 1000 iteration cycles. In all of the calculations presented here, we set $\mu^{*}\left(\omega_{c}\right)=0.13$ for the Coulomb pseudopotential with a cutoff frequency $\omega_{c}>0.5 \mathrm{eV}$. We have also checked that $\omega_{c}$ is sufficiently large and that results are well converged with this cutoff. The analytic continuation was performed numerically by employing the high-accuracy Padé scheme based on symbolic computation $[8,56]$ with a chosen precision of 250 decimal digits. After this procedure, we calculate the retarded momentum-dependent Green's function, the tunneling spectra, and the superconducting gap edge.
[1] H. Suhl, B. T. Matthias, and L. R. Walker, Phys. Rev. Lett. 3, 552 (1959).

[2] J. Nagamatsu, N. Nakagawa, T. Muranaka, Y. Zenitani, and J. Akimitsu, Nature (London) 410, 63 (2001).

[3] T. E. Weller, M. Ellerby, S. S. Saxena, R. P. Smith, and N. T. Skipper, Nat. Phys. 1, 39 (2005).

[4] M. Iavarone, G. Karapetrov, A. Koshelev, W. K. Kwok, D. Hinks, G. W. Crabtree, W. N. Kang, E.-M. Choi, H. J. Kim, and S.-I. Lee, Supercond. Sci. Technol. 16, 156 (2003).

[5] H. J. Choi, D. Roundy, H. Sun, M. L. Cohen, and S. G. Louie, Nature (London) 418, 758 (2002).

[6] D. Mou, R. Jiang, V. Taufour, S. L. Bud'ko, P. C. Canfield, and A. Kaminski, Phys. Rev. B 91, 214519 (2015).

[7] E. R. Margine and F. Giustino, Phys. Rev. B 87, 024505 (2013).

[8] A. Aperis, P. Maldonado, and P. M. Oppeneer, Phys. Rev. B 92, 054516 (2015).

[9] M. V. Milošević and A. Perali, Supercond. Sci. Technol. 28, 060201 (2015).

[10] V. Stanev and Z. Tešanović, Phys. Rev. B 81, 134522 (2010).

[11] E. Babaev, Phys. Rev. Lett. 89, 067001 (2002).

[12] J. Garaud, J. Carlström, and E. Babaev, Phys. Rev. Lett. 107, 197001 (2011).

[13] R. M. da Silva, M. V. Milošević, A. A. Shanenko, F. M. Peeters, and J. A. Aguiar, Sci. Rep. 5, 12695 (2015).

[14] L. Komendová, Y. Chen, A. A. Shanenko, M. V. Milošević, and F. M. Peeters, Phys. Rev. Lett. 108, 207002 (2012).

[15] M. Silaev and E. Babaev, Phys. Rev. B 85, 134514 (2012).

[16] N. V. Orlova, A. A. Shanenko, M. V. Milošević, F. M. Peeters, A. V. Vagov, and V. M. Axt, Phys. Rev. B 87, 134510 (2013).

[17] A. Linscheid, A. Sanna, A. Floris, and E. K. U. Gross, Phys. Rev. Lett. 115, 097002 (2015).
[18] P. Cudazzo, G. Profeta, A. Sanna, A. Floris, A. Continenza, S. Massidda, and E. K. U. Gross, Phys. Rev. Lett. 100, 257001 (2008).

[19] C. Bersier, A. Floris, A. Sanna, G. Profeta, A. Continenza, E. K. U. Gross, and S. Massidda, Phys. Rev. B 79, 104503 (2009).

[20] C. Brun, T. Cren, and D. Roditchev, Supercond. Sci. Technol. 30, 013003 (2017).

[21] T. Uchihashi, Supercond. Sci. Technol. 30, 013002 (2017).

[22] S. Qin, J. Kim, Q. Niu, and C. Shih, Science 324, 1314 (2009).

[23] T. Zhang, P. Cheng, W. Li, Y. Sun, G. Wang, X. Zhu, K. He, L. Wang, X. Ma, X. Chen, Y. Wang, Y. Liu, H. Lin, J. Jia, and Q. Xue, Nat. Phys. 6, 104 (2010).

[24] Y. Cao, A. Mishchenko, G. L. Yu, E. Khestanova, A. P. Rooney, E. Prestat, A. V. Kretinin, P. Blake, M. B. Shalom, C. Woods, J. Chapman, G. Balakrishnan, I. V. Grigorieva, K. S. Novoselov, B. A. Piot, M. Potemski, K. Watanabe, T. Taniguchi, S. J. Haigh, A. K. Geim, and R. V. Gorbachev, Nano Lett. 15, 4914 (2015).

[25] M. M. Ugeda, A. J. Bradley, Y. Zhang, S. Onishi, Y. Chen, W. Ruan, C. Ojeda-Aristizabal, H. Ryu, M. T. Edmonds, H.-Z. Tsai, A. Riss, S.-K. Mo, D. Lee, A. Zettl, Z. Hussain, Z.-X. Shen, and M. F. Crommie, Nat. Phys. 12, 92 (2016).

[26] X. Xi, Z. Wang, W. Zhao, J.-H. Park, K. T. Law, H. Berger, L. Forró, J. Shan, and K. F. Mak, Nat. Phys. 12, 139 (2015).

[27] G. Profeta, M. Calandra, and F. Mauri, Nat. Phys. 8, 131 (2012).

[28] D. M. Guzman, H. M. Alyahyaei, and R. A. Jishi, 2D Mater. 1, 021005 (2014).

[29] B. M. Ludbrook, G. Levy, P. Nigge, M. Zonno, M. Schneider, D. J. Dvorak, C. N. Veenstra, S. Zhdanovich, D. Wong, P. Dosanjh, C. Straßer, A. Stöhr, S. Forti, C. R. Ast, U. Starke, and A. Damascelli, Proc. Natl. Acad. Sci. 112, 11795 (2015). 
[30] K. Kanetani, K. Sugawara, T. Sato, R. Shimizu, K. Iwaya, T. Hitosugi, and T. Takahashi, Proc. Natl. Acad. Sci. 109, 19610 (2012).

[31] J. Chapman, Y. Su, C. A. Howard, D. Kundys, A. N. Grigorenko, F. Guinea, A. K. Geim, I. V. Grigorieva, and R. R. Nair, Sci. Rep. 6, 23254 (2016).

[32] N. I. Verbitskiy, A. V. Fedorov, C. Tresca, G. Profeta, L. Petaccia, B. V. Senkovskiy, D. Y. Usachov, D. V. Vyalikh, L. V. Yashina, A. A. Eliseev, T. Pichler, and A. Grüneis, 2D Mater. 3, 045003 (2016).

[33] A. T. Bollinger, G. Dubuis, J. Yoon, D. Pavuna, J. Misewich, and I. Božović, Nature (London) 472, 458 (2011).

[34] J. Ge, Z. Liu, C. Liu, C. Gao, D. Qian, Q. Xue, Y. Liu, and J. Jia, Nat. Mater. 14, 285 (2015).

[35] T. Golod, A. Iovan, and V. M. Krasnov, Nat. Commun. 6, 8628 (2015).

[36] F. Najafi, J. Mower, N. C. Harris, F. Bellei, A. Dane, C. Lee, X. Hu, P. Kharel, F. Marsili, S. Assefa, K. K. Berggren, and D. Englund, Nat. Commun. 6, 5873 (2015).

[37] P. J. Lowell, J. A. B. Mates, W. B. Doriese, G. C. Hilton, K. M. Morgan, D. S. Swetz, J. N. Ullom, and D. R. Schmidt, Appl. Phys. Lett. 109, 142601 (2016).

[38] R. Saniz, B. Partoens, and F. M. Peeters, Phys. Rev. B 87, 064510 (2013).

[39] H. Tang and S. Ismail-Beigi, Phys. Rev. B 80, 134113 (2009).

[40] C. Cepek, R. Macovez, M. Sancrotti, L. Petaccia, R. Larciprete, S. Lizzit, and A. Goldoni, Appl. Phys. Lett. 85, 976 (2004).

[41] K. Szałowski, Phys. Rev. B 74, 094501 (2006).

[42] A. V. Pogrebnyakov, J. M. Redwing, S. Raghavan, V. Vaithyanathan, D. G. Schlom, S. Y. Xu, Q. Li, D. A. Tenne, A. Soukiassian, X. X. Xi, M. D. Johannes, D. Kasinathan, W. E. Pickett, J. S. Wu, and J. C. H. Spence, Phys. Rev. Lett. 93, 147006 (2004).

[43] J.-C. Zheng and Y. Zhu, Phys. Rev. B 73, 024509 (2006).

[44] J. Pešić, R. Gajić, K. Hingerl, and M. Belić, Europhys. Lett. 108, 67005 (2014).
[45] C. Si, Z. Liu, W. Duan, and F. Liu, Phys. Rev. Lett. 111, 196802 (2013).

[46] C. Si, Z. Sun, and F. Liu, Nanoscale 8, 3207 (2016).

[47] X. Gonze, B. Amadon, P.-M. Anglade, J.-M. Beuken, F. Bottin, P. Boulanger, F. Bruneval, D. Caliste, R. Caracas, M. Côté, T. Deutsch, L. Genovese, P. Ghosez, M. Giantomassi, S. Goedecker, D. Hamann, P. Hermet, F. Jollet, G. Jomard, S. Leroux, M. Mancini, S. Mazevet, M. Oliveira, G. Onida, Y. Pouillon, T. Rangel, G.-M. Rignanese, D. Sangalli, R. Shaltaf, M. Torrent, M. Verstraete, G. Zerah, and J. Zwanziger, Comput. Phys. Commun. 180, 2582 (2009).

[48] S. Y. Savrasov and D. Y. Savrasov, Phys. Rev. B 54, 16487 (1996).

[49] J. Bekaert, S. Vercauteren, A. Aperis, L. Komendová, R. Prozorov, B. Partoens, and M. V. Milošević, Phys. Rev. B 94, 144506 (2016).

[50] C.-Y. Moon, Y.-H. Kim, and K. J. Chang, Phys. Rev. B 70, 104522 (2004).

[51] We note that this result is different from that obtained in Ref. [57] for two-ML $\mathrm{MgB}_{2}$, where $T_{\mathrm{c}}$ was found to exceed the bulk value. The difference can be traced back to the unreasonably low Coulomb pseudopotential used in this work to compensate the lack of multiband effects in their isotropic Eliashberg approach.

[52] S. Souma, Y. Machida, T. Sato, T. Takahashi, H. Matsui, S.-C. Wang, H. Ding, A. Kaminski, J. C. Campuzano, S. Sasaki, and K. Kadowaki, Nature (London) 423, 65 (2003).

[53] G. Grimvall, The Electron-Phonon Interaction (North Holland, Amsterdam, 1981).

[54] We note that for compressive strains exceeding $-1.5 \%, \sigma$ and $S$ gaps become hybridized, albeit their contributions can still be distinguished. Their partial overlap is not due to new physics-it is provoked by a general depletion of the superconducting gap values, forcing the gaps closer together.

[55] D. R. Hamann, Phys. Rev. B 88, 085117 (2013).

[56] K. S. D. Beach, R. J. Gooding, and F. Marsiglio, Phys. Rev. B 61, 5147 (2000).

[57] T. Morshedloo, M. Roknabadi, and M. Behdani, Physica C Supercond. 509, 1 (2015). 